\title{
BOUNDED SURGERY \\ AND DIHEDRAL GROUP ACTIONS ON SPHERES
}

\author{
IAN HAMBLETON AND ERIK KJÆR PEDERSEN
}

\section{CONTENTs}

1. Introduction

2. Topological representations

3. Controlled algebra and topology

4. Mackey properties

5. Infinite group actions and topological representations

6. The transfer

7. The obstruction

8. Actions on $S^{n} \times R^{m}$

\section{INTRODUCTION}

If a finite group $G$ acts freely and simplicially on a complex homotopy equivalent to a sphere $S^{n}$, then $G$ has periodic Tate cohomology: $H^{i+n+1}(G ; Z) \cong$ $H^{i}(G ; Z)$ for all $i>0$. Swan proved in [S2] that this condition was also sufficient. For free topological actions on $S^{n}$ itself, the first additional restriction is:

Theorem [Mi]. A finite dihedral group does not act freely and topologically on $S^{n}$.

Milnor's argument used the compactness of $S^{n}$ as well as the manifold structure. In fact, for dihedral groups with periodic cohomology, i.e., of order $2 n$

Received by the editors March 5, 1990.

1980 Mathematics Subject Classification (1985 Revision). Primary 57S30, 57S17, 18 F25.

Key words and phrases. Discontinuous group actions, crystallographic groups, surgery obstructions. 
where $n$ is odd, we have,

Example. Any finite dihedral group acts freely and smoothly on $S^{3} \times R^{3}$.

The proof follows directly from the existence of a smooth normal invariant for the Swan complex $X$ associated to the given dihedral group [Ma]. A surgery problem

$$
f \times 1: M \times\left(D^{3}, S^{2}\right) \longrightarrow X \times\left(D^{3}, S^{2}\right)
$$

is normally cobordant to a homotopy equivalence of pairs by the $\pi$ - $\pi$-theorem. The interior of the resulting manifold gives the example.

Another attempt to generalize the problem is to study actions on a sphere $S^{n+k}$ with a standardly embedded invariant subsphere $S^{k-1}$. This situation is related to the last one if we assume that the action is free away from the $S^{k-1}$, since $S^{n+k}-S^{k-1} \approx S^{n} \times R^{k}$.

Theorem [AP,HM1]. If $n \geq 1$, a finite dihedral group does not act semifreely and topologically on $S^{n+k}$ with fixed standard subsphere $S^{k-1}$.

Note that a free action on $S^{n} \times R^{k}$ must converge to the trivial action on "the $S^{k-1}$ at $\infty$ " in order to be extended to a semifree action on $S^{n+k}$. More generally we study free actions on $S^{n} \times R^{k}$ that converge to a given action on the $S^{k-1}$ at $\infty$.

In this paper we study such actions where the action at infinity is given by a real linear representation, and relate such actions to a question of Wall [W4, p. 518]: "it is natural to ask whether any countable group with periodic Farrell cohomology can act freely and properly on some product $S^{n} \times R^{k}$ or whether a condition such as Milnor's is necessary. I observe at least that it is not necessary for each element of order 2 to be in the centre." Wall then shows that the infinite dihedral group acts freely and properly discontinuously on $S^{2} \times R$ with compact quotient.

Connolly and Prassidis [CP] showed that any group $\Gamma$ with finite virtual cohomological dimension and periodic Farrell cohomology acts freely and properly discontinuously, with non-compact orbit space. A remaining question is: for actions with compact quotient, can $\Gamma$ contain a finite dihedral subgroup?

Denoting the nontrivial one-dimensional representation by $R_{-}$, we prove

Theorem 7.11. Let $V$ be a linear representation of the dihedral group of order $2 p, D_{p}, p$ an odd prime. Then there is a topological action of $D_{p}$ on a sphere free off a standard proper subsphere and given by the unit sphere $S V$ on the subsphere if and only if the representation has at least two $R_{-}$-factors.

However Milnor's condition is not necessary in general since

Theorem 8.3. The group $D_{p} \times_{\alpha} Z^{k}$ acts freely and properly discontinuously on $S^{n} \times R^{m}$ for some $n, m$ with compact quotient if and only if $n \equiv 3(4), m=k$, and $\alpha$ considered as a real representation has at least two $R_{-}$-factors.

Remark. We prove our nonexistence results by using 7.11 so the nonexistence results in Theorem 8.3 concern topological actions. On the other hand, the ac- 
tions constructed in Theorem 8.3 are actually smooth. The lowest dimensional example is a cocompact action on $S^{3} \times R^{2}$.

The authors would like to thank Tom Farrell and Frank Connolly for useful discussions.

\section{TOPOLOGICAL REPRESENTATIONS}

In this section $G$ is a finite group. The unit spheres in linear representations can be thought of as models for smooth group actions. In the homotopy category the concept "homotopy representation" has been introduced by Tammo tom Dieck, meaning a group acting on a CW complex, so that the fixed set of each subgroup is homotopy equivalent to a sphere.

Topological group actions can be extremely wild, but we want to consider the simplest possible class, which is still sufficiently big to contain interesting non-PL examples.

Definition 2.1. A topological representation is a group action of $G$ on a sphere $S^{n}$ such that the fixed set of a subgroup $H,\left(S^{n}\right)^{H}$, is a linear subsphere of $S^{n}$.

Example 2.2. tom Dieck and Löffler construct actions of $C_{p} \times C_{p}, C_{p}$ the cyclic group of order $p$ on $S^{2 n+1}$ where the fixed sets of the two $C_{p}$ 's are locally flat $n$-spheres, that are linked with linking number different from 0 [DL]. These are smoothings of homotopy representations, but they are not topological representations by our definitions.

It is clear that the join of two topological representations is again a topological representation, so we may form the Grothendieck construction with join as the sum to get a topological representation group. We do not get a ring even if we allow actions that are not effective, since there is no obvious analogue of the tensor product construction for linear representations. In fact Steffen Bentzen [B] has shown there are finite groups $G_{1}$ and $G_{2}$ of coprime order, so that $G_{1}$ acts freely on $S^{2 k-1}$ and $G_{2}$ acts freely on $S^{2 l-1}$ but such that $G_{1} \times G_{2}$ has no free action on $S^{2 k l-1}$.

In this paper we shall study a specific kind of topological representations, that are free off a given subrepresentation.

We shall call a finitely dominated complex a Swan complex if it has finite fundamental group and the universal cover is homotopy equivalent to a sphere [S2].

Proposition 2.3. If $\left(S^{n+k}, G\right)$ is a topological representation which is free away from a representation $\left(S^{k-1}, G\right)$, then $\left(S^{n+k}-S^{k-1}\right) / G$ is a Swan complex, hence $G$ is a periodic group.

Proof. $\left(S^{n+k}-S^{k-1}\right) / G$ is finite dimensional and the universal cover is of the homotopy type of a sphere. It is proved in [E] that $\left(S^{n+k}-S^{k-1}\right) / G$ is finitely dominated. 
Assume $G$ acts on the topological space $X$ and by isometries on the metric space $M$.

Definition 2.4. A map $p$ from $X$ to $M$ is called almost equivariant if there exists $k$ so that $d(p g x, g p x)<k$.

If the homotopy type of $\left(S^{n+k}-S^{k-1}\right) / G$ is denoted by $X$, then $X$ is determined by $G$ and one $k$-invariant. We may consider the induced action of $G$ on $R^{k}$ (the open cone of the action on $S^{k-1}$ ), and we obtain a map $\widetilde{X} \times{ }_{G} R^{k} \rightarrow R^{k} / G$, which is a bounded Poincare duality complex in the sense of [FP2]. We may thus consider the bounded structure set $\mathscr{S}^{b}\left(\widetilde{X} \times_{G} R^{k} \rightarrow R^{k} / G\right)$, an element of which is a bounded homotopy equivalence $W \rightarrow \widetilde{X} \times{ }_{G} R^{k}$. The universal covering of $W$ is an element of $\mathscr{S}^{b}\left(S^{n} \times R^{k} \stackrel{p}{\rightarrow} R^{k}\right)$, where $p$ is projection on the second factor, but it follows from [FP2] that this set only contains one element, so there is a bounded homotopy of $\widetilde{W} \rightarrow S^{n} \times R^{k}$ to a homeomorphism. It follows that $G$ acts on $S^{n} \times R^{k}$ and $p$ is almost equivariant. Choosing a radial homeomorphism of $R^{k} \rightarrow \dot{D}^{k}$, the open unit disk, we get $S^{n} \times \dot{D}^{k} \rightarrow \dot{D}^{k}$. If we now give $\left(S^{n}, x\right)$ a metric which is $1-\|x\|$ times the standard metric, the metric completion of $S^{n} \times \dot{D}^{k}$ is easily seen to be $S^{n+k}$ (the join of $S^{n}$ and $S^{k-1}$ ). When the metric is changed this way, a bounded subset near infinity in $S^{n} \times R^{k}$ becomes small near $S^{k-1}$. The group action thus extends continuously to $S^{n+k}=\left(S^{n} \times R^{k}\right) \cup S^{k-1}$ because $p$ is almost equivariant. This method was used in [AP] in the case where the action on $S^{k-1}$ is trivial, and the general principle goes back to [AH].

Theorem 2.5. There is a 1-1 correspondence between the bounded structure set $\mathscr{S}^{b}\left(\widetilde{X} \times_{G} R^{k}\right)$ and conjugacy classes of group actions on $S^{n+k}$ which are the given action on the standard subsphere $S^{k-1}$ and where the homotopy type of $\left(S^{n+k}-S^{k-1}\right) / G$ is $X$.

Proof. We have shown how to get from an element of $\mathscr{S}^{b}\left(\widetilde{X} \times{ }_{G} R^{k}\right)$ to a group action on $S^{n+k}$, so assume $G$ acts on $S^{n+k}=S^{n} * S^{k-1}$, free off $S^{k-1}$ and by $\alpha$ on $S^{k-1}$. There is a map $p: S^{n+k}=S^{n} * S^{k-1} \rightarrow 0 * S^{k-1}$, where 0 is the origin of $R^{k}$ and thus $0 * S^{k-1}$ is $D^{k}$, the unit disk. Letting $G$ act on $D^{k}$ by $\alpha, p$ is equivariant when restricted to $S^{k-1}$. Consider

$$
\tilde{p}(x)=\frac{1}{|G|} \sum_{g \in G} g^{-1} p(g x),
$$

where the sum is taking place in $R^{k}$. Clearly $\tilde{p}$ is equivariant. Also $\tilde{p} \mid S^{k-1}=$ $p \mid S^{k-1}$ and $\tilde{p}\left(S^{n+k}-S^{k-1}\right)$ is the interior of $D^{k}$. The distance between $\tilde{p}(x)$ and $p(x)$ is thus a function that goes to 0 as $x$ approaches $S^{k-1}$. We now choose a radial and hence equivariant identification of $\dot{D}^{k}$ with $R^{k}$ and $S^{n+k}-$ $S^{k-1}$ with $S^{n} \times R^{k} \quad\left((\bar{x}, t \bar{v})\right.$ in $S^{n} \times R^{k}$ corresponds to the join line from 
$\bar{x} \in S^{n}$ to $\bar{v} \in S^{k-1}$ ). Since the distance between $p$ and $\tilde{p}$ goes to 0 as we approach $S^{k-1}$, this may be done so that the distance between $p$ and $\tilde{p}$ in $R^{k}$ is bounded. Letting $X=\left(S^{n} \times R^{k}\right) / G$, we get the following diagram:

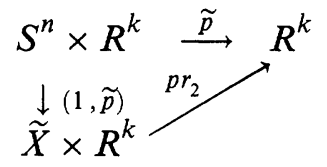

where all the maps are equivariant. We want to show $(1, \tilde{p})$ is an equivariant bounded homotopy equivalence. Using [AM1] it is enough to show it is a bounded homotopy equivalence, but this follows from $\widetilde{p}$ being close to $p$, the projection. This shows the map is onto. To see it is monic, we need that radial reparametrization induces the identity on the structure set [FP2].

\section{CONTROLled ALGeBRA AND TOPOLOGY}

Most of the results of this section will appear in [FP2, FP1, R2]. Let $M$ be a metric space. Assume there is a group $G$ acting on $M$ by eventual Lipschitz maps [PW]. Recall that an eventual Lipschitz map $g: M \rightarrow M$ is a map, so there exists $k, l \in R_{+}$such that $d(g x, g y) \leq k \cdot d(x, y)+l$. We want $k$ and $l$ to be independent of $g$.

Remark 3.1. The affine function $z \rightarrow k \cdot z+l$ may actually be replaced by any function sending $R_{+}$monically onto $R_{+}$as has been observed by J. Rosenberg [Ro]. We want this function to be independent of $g \in G$. This is of course only a problem when $G$ is infinite.

Example 3.2. Let $M$ be a finitely generated group exhibited with the word metric, and $G \subseteq M$ a subgroup. Then the action of $G$ on $M$ by conjugation is by eventual Lipschitz equivalences. Specifically, if $g \in G$ has length $l$ then $d\left(g x g^{-1}, g y g^{-1}\right)=d\left(g x y^{-1} g^{-1}, e\right) \leq 2 l+d(x, y)$

Example 3.3. Let $(V, G)$ be an orthogonal representation. Then $G$ acts by isometries on $V$, hence clearly by eventual Lipschitz maps.

Given $M$ and $G$ as above, and a commutative ring with unit $R$, we define a category $\mathscr{G}_{M, G}(R)$ as follows:

Definition 3.4. An object $A$ is a left $R(G)$-module together with a map $f: A \rightarrow$ $F(M)$, where $F(M)$ is the set of finite subsets of $M$, satisfying the following conditions:

(i) $f$ is $G$-equivariant.

(ii) $A_{x}=\{a \in A \mid f(a) \subseteq\{x\}\}$ is a finitely generated free sub- $R$-module.

(iii) As an $R$-module, $A=\bigoplus_{x \in M} A_{x}$.

(iv) $f(a+b) \subseteq f(a) \cup f(b)$.

(v) For each ball $B \subset M\left\{x \in B \mid A_{x} \neq 0\right\}$ is finite.

A morphism $\phi: A \rightarrow B$ is a morphism of $R G$-modules satisfying the following condition: there exists $k$ so that the components $\phi_{n}^{m}: A_{m} \rightarrow B_{n}$ (which are 
$R$-module morphisms) are zero when $d(m, n)>k . \mathscr{G}_{M, G}(R)$ is an additive category in an obvious way.

Remark 3.5. It follows easily from the conditions that $f$ measures exactly where an element has components, i.e., if $x_{1}, \ldots, x_{n} \in M$ are different points and $a_{i} \in A_{x_{i}}, a_{i} \neq 0$, then $f\left(a_{1}+\cdots+a_{n}\right)=\left\{x_{1}, \ldots, x_{n}\right\}$.

Given an object $A$, an $R$-module homomorphism $\phi: A \rightarrow R$ is said to be locally finite if the set of $x \in M$ for which $\phi\left(A_{x}\right) \neq 0$ is finite. Define $A^{*}=\operatorname{Hom}_{R}^{1 . f .}(A, R)$, the set of locally finite $R$-homomorphisms. We want to make * a functor from $\mathscr{G}_{M, G}(R)$ to itself to make $\mathscr{G}_{M, G}(R)$ a category with involution. We define $f^{*}: A^{*} \rightarrow F M$ by $f^{*}(\phi)=\left\{x \mid \phi\left(A_{x}\right) \neq 0\right\}$, which is finite by assumption. $A^{*}$ has an obvious right action of $G$, turning it into a right $R G$ module given by $\phi g(a)=\phi(g a)$, and $f^{*}$ is equivariant with respect to the right action on $M$ given by $x g=g^{-1} x$. To make $*$ an endofunctor of $\mathscr{G}_{M, G}(R)$ we need to replace the right action by a left action. As is usual in surgery theory, this may be done in various ways, the standard one being to let $g$ act on the left by letting $g^{-1}$ act on the right. However given a homomorphism $w: G \rightarrow\{ \pm 1\}$, we may let $g$ act on the left of $A^{*}$ by letting $w(g) \cdot g^{-1}$ act on the right. We also obtain the generalization of geometric antistructures as follows: Given a group automorphism $\theta_{G}: G \rightarrow G$ and an eventual Lipschitz equivalence $\theta_{M}: M \rightarrow M$ so that $\theta_{M}(g \cdot m)=\theta_{G}(g) \cdot \theta_{M}(m)$, satisfying $\theta_{M}^{2}(m)=b m$ and $\theta_{G}^{2}(g)=b g b^{-1}$ for some $b \in G$, we may then twist the involution $*$ by composing with the functor sending $(A, f)$ to $\left(A^{\theta}, f^{\theta}\right)$, where $A^{\theta}$ is the same $R$-module, but $g$ acts on the left by multiplication by $\theta(g)$ and $f^{\theta}=\theta_{M}^{-1} \cdot f$.

If $\theta_{G}$ is an inner automorphism, this is just scaling and induces an isomorphism of $L$-groups. We are thus essentially allowing antistructures of the type Hambleton, Taylor, and Williams [HTW1] call "geometric" antistructures, with the further restriction that there has to be a counterpart at the metric space level.

Remark 3.6. It is tempting to allow an arbitrary additive category $\mathscr{A}$ in place of just the category of f.g. free $R$-modules in the definition of $\mathscr{G}_{M, G}(R)$. This is indeed possible, but we do not need it for the purpose of this paper. An easy check demonstrates

Proposition 3.7. $\left(\mathscr{G}_{M, G}(R), *\right)$, where * is among the above suggestions, is an additive category with involution in the sense of Ranicki [R1].

For many purposes we are more interested in the subcategory of $\mathscr{G}_{M, G}(R)$ for which all objects are free $R(G)$-modules.

Definition 3.8. The subcategory of $\mathscr{G}_{M, G}(R)$, where the modules are required to be free $R(G)$-modules, is denoted by $\mathscr{C}_{M, G}(R)$.

It is easy to see that $*$ induces a functor on $\mathscr{C}_{M, G}(R)$, so that $\mathscr{C}_{M, G}(R)$ is a subcategory with involution. 
Example 3.9. If $G$ acts trivially on $M$ and $G$ is finite, then $\mathscr{C}_{M, G}(R)$ is naturally equivalent to $\mathscr{C}_{M}(R G)$, where $R G$ is the category of free finitely generated based $R G$-modules.

Example 3.10. If $G$ is finitely generated and $|G|$ denotes the metric space with the same underlying set as $G$, and the word metric, then $\mathscr{C}_{|G|, G}(R)$ is naturally equivalent to $\mathscr{C}_{p t}(R G)$ (as categories with involution). Notice it does not matter which generating set we choose for $G$ since two different generating sets will give eventual Lipschitz equivalent metrics. In case $G$ is finite, this means $\mathscr{C}_{|G|, G}(R)$ is equivalent to $\mathscr{C}_{p t, G}(R)$, which is equivalent to $\mathscr{C}_{p t}(R G)$.

Using the algebraic $L$-theory of additive categories with involution, we immediately have defined functors $L_{n}^{K}\left(\mathscr{C}_{M, G}(R)\right)$, where $K$ is some $*$ invariant subgroup of $\widetilde{K}_{i}\left(\mathscr{C}_{M, G}(R)\right), i=0,1$. Here $\widetilde{K}_{1}\left(\mathscr{C}_{M, G}(R)\right)=K_{1}\left(\mathscr{C}_{M, G}(R)\right) /\{ \pm 1\}$ and $\widetilde{K}_{0}\left(\mathscr{C}_{M, G}(R)\right)=\left(K_{0}\left(\mathscr{C}_{M, G}(R)\right)^{\wedge}\right) / K_{0}\left(\mathscr{C}_{M, G}(R)\right)$, where ${ }^{\wedge}$ denotes idempotent completion.

Let $N$ be a submetric space of the metric space $M$. In the equivariant case, we suppose that $N$ is an invariant subspace.

Definition 3.11. The category $\mathscr{C}_{M, G}^{>N}(R)$ of germs away from $N$ has the same objects as $\mathscr{C}_{M, G}(R)$, and morphisms are germs of morphisms away from $N$, i.e., two morphisms are identified if there exists $k$ so that they only differ in a $k$-neighborhood of $N$.

Consider the metric space $M \times R$, where $G$ acts trivially on the $R$-factor. Inside we have the metric space $M \cup N \times[0, \infty)$. It follows immediately from the methods of [PW] (see also [AM2] for a more formalized description) that the natural functor

$$
\mathscr{C}_{M \cup N \times[0, \infty), G}(R) \longrightarrow \mathscr{C}_{M, G}^{>N}(R)
$$

induces an isomorphism on $K$-theory, and it follows from the proofs of [R2] that it induces an isomorphism in $L$-theory (Eilenberg swindle is allowed in $L$-theory). Hence from [PW] we get a long exact sequence

$$
\cdots K_{*}\left(\mathscr{C}_{M, G}(R)\right) \longrightarrow K_{*}\left(\mathscr{C}_{M, G}^{>N}(R)\right) \longrightarrow K_{*-1}\left(\mathscr{C}_{N, G}(R)\right) \cdots
$$

Here it should be noted that we are using the nonconnective deloopings of [PW] to define $K$-theory in negative dimensions. The following is essentially proved by Ranicki [R2]

Theorem 3.12. There is a 4-periodic long exact sequence

$$
\begin{aligned}
& \cdots L_{n}^{h}\left(\mathscr{C}_{(N, G)}(R)\right) \longrightarrow L_{n}^{h}\left(\mathscr{C}_{(M, G)}(R)\right) \\
& \quad \longrightarrow L_{n}^{K}\left(\mathscr{C}_{(M, G)}^{>N}(R)\right) \longrightarrow L_{n-1}^{h}\left(\mathscr{C}_{(N, G)}(R)\right) \cdots,
\end{aligned}
$$

where $K=\operatorname{Im}\left(\widetilde{K}_{1}\left(\mathscr{C}_{(M, G)}(R)\right) \rightarrow \widetilde{K}_{1}\left(\mathscr{C}_{(M, G)}^{>N}(R)\right)\right)$.

The formulation in [R2] uses $\mathscr{C}_{M \cup N \times[0, \infty)}$ instead of $\mathscr{C}_{M}^{>N}$. 
We saw in Example 3.9 that trivial group action corresponds to $R G$ coefficients. This is part of a more general phenomenon motivating the following definition.

Definition 3.13. Suppose $G$ is acting on the metric space $M$ with invariant subspace $N$. We say that the set of subgroups $\left\{H_{\alpha}\right\}$ of $G$ is the effective fundamental group for $(M, G)$ away from $N$ if the following is satisfied: For every $k>0$ the set $\left\{x \in M \mid \operatorname{diam}\left(H_{\alpha} \cdot x\right)<k\right\}$ is not contained in a bounded neighborhood of $N$.

Example 3.14. Let $(V, G)$ be a representation. Then the effective fundamental group away from 0 is the set of isotropy subgroups of the representation.

On the geometric side we need the following result from [FP2]. A map $X \rightarrow M$ from a space to a metric space is eventually continuous if there exist a covering $\left\{U_{\alpha}\right\}$ of $X$ so that $\operatorname{diam}\left(p U_{\alpha}\right)$ is uniformly bounded, and the inverse image of a bounded set is precompact. When the metric space is a cone, an eventually continuous map may always be replaced by a continuous map which is only a bounded distance away.

Theorem 3.15. Let $X$ be a free $G-C W$ complex together with a G-equivariant eventually continuous map $X \rightarrow M$ such that $X \rightarrow M$ is boundedly simply connected, and $X$ satisfies Poincaré duality with respect to some homomorphism $w: G \rightarrow Z / 2$ in the category $\mathscr{C}_{M, G}(Z), \operatorname{dim}(X) \geq 5$. Let $W \rightarrow X$ be a degree-one normal map. Then $W$ is normally cobordant to a bounded homotopy equivalence if and only if an invariant in $L_{n}\left(\mathscr{C}_{M, G}(Z)\right)$ vanishes.

The concept boundedly simply connected is defined in [FP2, 2.7]. As in standard surgery theory, normal invariants correspond to lifts of the Spivak normal fibre space $X \rightarrow B F$ to $B T O P$. If we fix a lift (defining a basepoint) then [FP2]:

Theorem 3.16. There is a long exact sequence of surgery

$$
\begin{aligned}
\cdots & \left.\longrightarrow L_{n+1}^{h} \mathscr{C}_{M, G}(Z)\right) \longrightarrow \mathscr{S}^{b}\left(\begin{array}{c}
X / G \\
\downarrow \\
M / G
\end{array}\right) \\
& \longrightarrow[X / G, F / T O P] \longrightarrow L_{n}^{h}\left(\mathscr{C}_{M, G}(Z)\right) .
\end{aligned}
$$

Tensor product defines a pairing

$$
\mathscr{C}_{|G|, G}(R) \times \mathscr{G}_{M, G}(R) \longrightarrow \mathscr{C}_{|G| \times M, G}(R)
$$

whenever $R$ is a commutative ring with unit. When $|G|$ is finite, this means we may replace $\mathscr{C}_{|G|, G}(R)$ by $\mathscr{C}_{p t}(R G)$ and $\mathscr{C}_{|G| \times M, G}(R)$ by $\mathscr{C}_{M, G}(R)$, so for finite $G$ we have a pairing

$$
\mathscr{C}_{p t}(R G) \times \mathscr{G}_{M, G}(R) \longrightarrow \mathscr{C}_{M, G}(R) .
$$


Using the fact that $(A \otimes B)^{*}=A^{*} \otimes B^{*}$ for finitely generated $R$-modules, it follows that this commutes with the pairings, so it follows from [R1] that there is a pairing

$$
L_{n}(R G) \otimes L^{k}\left(\mathscr{G}_{M, G}(R)\right) \longrightarrow L_{n+k}\left(\mathscr{C}_{M, G}(R)\right)
$$

geometrically corresponding to the twisted product.

\section{MACKEY PROPERTIES}

Let $M$ be a metric space, $G$ a finite group acting on $M$ by eventual Lipschitz maps, and $R$ a commutative ring with unit. Consider the category $\mathscr{C}_{M, G}(R)$. Given two subgroups $G_{1} \subset G_{2} \subset G$ we have $G_{1}$ and $G_{2}$ acting on $M$ by restriction, restriction functors $\mathscr{C}_{M, G_{2}}(R) \rightarrow \mathscr{C}_{M, G_{1}}(R)$, and induction functors $\mathscr{C}_{M, G_{1}}(R) \rightarrow \mathscr{C}_{M, G_{2}}(R)$. The restriction functor is obtained just by restriction of the group action, and the induction functor sends an object $A$ to $R G_{2} \otimes_{R G_{1}} A$. The required map from $R G_{2} \otimes_{R G_{1}} A$ to the finite subsets of $M$ is extended from the map of $A$ to the finite subsets of $M$ by equivariance, i.e., by $f(g \otimes a)=$ $g \cdot f(a)$. Clearly restriction and induction are functors. We need

Lemma 4.1. Restriction and induction are functors of categories with involution. Proof. The involution is given by $A^{*}=\operatorname{Hom}^{1 . f .}(A, R)$ turned into a left $R G$ module as in $\S 3$, and it does not matter whether we restrict before or after applying $\mathrm{Hom}^{\text {l.f. }}$. Also

$$
\begin{aligned}
\operatorname{Hom}^{\text {l.f. }}\left(R G_{2} \otimes_{R G_{1}} A, R\right) & =\operatorname{Hom}\left(R G_{2}, \operatorname{Hom}^{\text {l.f. }}(A, R)\right) \\
& =R G_{2} \otimes_{R G_{1}} \operatorname{Hom}^{\text {l.f. }}(A, R) .
\end{aligned}
$$

Given two functors between additive categories with involution, we may form a new functor, the direct sum of the two functors. It is easy to see that

Lemma 4.2. A functor between additive categories with involution induces a map of L-groups. The sum of two functors induces the sum of the two maps.

Proof. The proof follows directly from the definitions since $L$-groups are defined as a bordism theory, where direct sum is turned into addition [R1].

Consider the category $A(G)$ defined as follows. The objects are the subgroups of $G$, and $\operatorname{Hom}\left(H_{1}, H_{2}\right)$ is the Grothendieck construction applied to the collection of finite "free bisets" (i.e., finite sets $Z$ with free left $\mathrm{H}_{2}$-action and free right $H_{1}$-action), where the addition is disjoint union. The balanced product

$$
\left({ }_{H_{3}} Z_{H_{2}}\right) \times{ }_{H_{2}}\left({ }_{H_{2}} Y_{H_{1}}\right)
$$

is a free biset and can be easily shown to induce a composition $\operatorname{Hom}\left(H_{1}, H_{2}\right) \times$ $\operatorname{Hom}\left(H_{2}, H_{3}\right) \rightarrow \operatorname{Hom}\left(H_{1}, H_{3}\right)$, which is bilinear. The set $H$ as an $H-H$ biset is the identity element for $\operatorname{Hom}(H, H)$.

There is a functor $\operatorname{Gr}(G) \rightarrow A(G)$ from the category of subgroups of $G$ (morphisms are $\operatorname{Maps}\left(H_{1}, H_{2}\right)=\left\{g \in G \mid g H_{1} g^{-1} \subset H_{2}\right\}$ ). It is the identity 
on the objects and sends $g \in \operatorname{Maps}\left(H_{1}, H_{2}\right)$ to the equivalence class of $H_{2}$ considered as a left $H_{2}$ set in the obvious manner, and $h_{2} h_{1}=h_{2} g h_{1} g^{-1}$ for all $h_{1} \in H_{1}$ and all $h_{2} \in H_{2}$. As noted in [HTW2, 4.1], this is a Mackey functor and any functor out of $A(G)$ to an additive category yields a Mackey functor by composition. It follows that

Theorem 4.3. Given a finite group $G$ and a metric space $M$ as above, then $\mathscr{C}_{M,-}(R)$ is a Mackey functor, and hence $L_{n}\left(\mathscr{C}_{M,-}(R)\right)$ is a Mackey functor.

Remark. We suppress the upper index in the $L$-groups in the above statement. The point is that the upper index has to be a subgroup of a $K$-theoretic group which is in itself a Mackey functor, e.g., the whole group or the trivial subgroup, but also naturally defined image groups will work.

Proof. Assume given an $H_{1}-H_{2}$ biset $Z$. Then sending $A$ to $R Z \otimes_{R H_{2}} A$ and extending the reference map by equivariance defines a functor from $\mathscr{C}_{X, H_{2}}(R)$ to $\mathscr{C}_{X, H_{1}}(R)$. The result now follows as described above from [HTW2].

By tensoring $L_{n}\left(\mathscr{C}_{M,-}(R)\right)$ with $\widehat{Z}_{2}$, we obtain a "2-local Mackey functor" on $\operatorname{Gr}(G)$. Let $\mathscr{O}_{\text {odd }}(\dot{G})$ denote the set of proper subgroups of $G$ with odd index.

The following result was proved in [HM1, 6.13].

Theorem 4.4. If $G=C_{d} \rtimes P$ is a 2-hyperelementary group ( $d$ is odd and $P$ is a 2-group) then any 2-local Mackey functor $\mathscr{M}$ has a natural splitting

$$
\mathscr{M}=\bigoplus\{\mathscr{M}(G)(l): l \mid d\}
$$

induced by idempotents in the 2-local Burnside ring. The summand $\mathscr{M}(G)(d)$ associated to the divisor $l=d$ is the kernel of the restriction maps

$$
\mathscr{M}(G) \longrightarrow \bigoplus\left\{\mathscr{M}(H) \mid H \subseteq G, H \in \mathscr{O}_{\text {odd }}(G)\right\} .
$$

The sum of the induction maps

$$
\bigoplus\left\{\mathscr{M}(H) \mid H \in \mathscr{O}_{\text {odd }}(G)\right\} \rightarrow \mathscr{M}(G)
$$

has image $\bigoplus\{\mathscr{M}(G)(l)|l \neq d, l| d\}$.

We refer to the component $\mathscr{M}(G)(d)$ as the "top component" of $\mathscr{M}(G)$.

Let $(K, L)$ be a $G-C W$ pair equivariantly embedded in a representation sphere $S^{n}$. Then there is an induced action on the metric space

$$
\left.\left.O(K)=0 \cup\left\{t \cdot x \in R^{n+1} \mid x \in K, t \in\right] 0, \infty\right)\right\} .
$$

Theorem 4.5. Let $G=C_{d} \rtimes P$ be a 2-hyperelementary group acting on $M=$ $O(K)$ with invariant $N=O(L)$. Suppose that the effective fundamental group for $(M, G)$ away from $O(L)$ is $\left\{H_{\alpha}\right\}$, where each $H_{\alpha} \subseteq H_{\alpha}^{\prime}$ for some $H_{\alpha}^{\prime} \in$ $\mathscr{O}_{\text {odd }}(G)$. Then

$$
L_{n}\left(\mathscr{C}_{N, G}(R)\right) \otimes \hat{Z}_{2} \cong L_{n}\left(\mathscr{C}_{M, G}(Z)\right) \otimes \widehat{Z}_{2}
$$

in the top component. 
Proof. The proof is by induction on cells, so assume $K$ is obtained from $L$ by attaching exactly one equivariant $k$-cell $D^{k} \times G / H$. Our assumption is $H \subseteq H^{\prime}$, where $H^{\prime}$ is a proper odd index subgroup. In view of Theorem 3.12 it is enough to prove that $L_{n}\left(\mathscr{C}_{M, G}^{>N}(R)\right) \otimes \widehat{Z}_{2}$ is trivial on the top component. But $O(M)-O(N)=\left(O\left(D^{k}\right)-O\left(S^{k-1}\right)\right) \times G / H$, so $\mathscr{C}_{M, G}^{>N}(R)$ is induced from $\mathscr{C}_{O\left(D^{k}\right) \times H^{\prime} / H, H^{\prime}}(R)$. It follows from Theorem 4.4 that $K_{*}\left(\mathscr{C}_{M, G}^{>N}(R)\right) \otimes \widehat{Z}_{2}$ and $L_{n}\left(\mathscr{C}_{M, G}^{>N}(R)\right) \otimes \widehat{Z}_{2}$ are trivial on the top component.

Remark 4.6. An important special case of Theorem 4.5 is when $L$ is empty, so $O(L)=(0)$, a point.

\section{INFINITE GROUP ACTIONS AND TOPOLOGICAL REPRESENTATIONS}

In this section we establish a connection between proper discontinuous free, cocompact actions of $G=D \times{ }_{\alpha} Z^{k}$ on a sphere crossed with euclidean space, $S^{n} \times R^{m}$, and topological representations of the finite group $D$. The group $D$ is assumed to be a periodic group; our example of main interest is $D$, the dihedral group of order $2 p, p$ a prime. We start by making a couple of observations:

Lemma 5.1. The sphere dimension $n$ has to be equivalent to $3 \bmod 4$.

Proof. This is the same as in Proposition 2.3.

Lemma 5.2. The euclidean space factor must have dimension $k$, i.e., $k=m$.

Proof. Choose $Z^{k} \subset D \times_{\alpha} Z^{k}$, a normal cofinal subgroup. By passing to a subgroup of index 2 if necessary, we may assume that $Z^{k}$ acts orientably. Now $\left(S^{n} \times R^{m}\right) / Z^{k}$ is an orientable manifold of dimension $n+m$, and there is a fibration

$$
S^{n} \times R^{m} \longrightarrow\left(S^{n} \times R^{m}\right) / Z^{k} \longrightarrow T^{k},
$$

so a homological consideration shows $k=m$.

By abuse of notation we shall use $\alpha$ to denote the real representation induced by the integral representation $\alpha$. We now prove the following

Theorem 5.3. Suppose that $D \times{ }_{\alpha} Z^{k}$ acts freely, cocompactly, and properly discontinuously on $S^{n} \times R^{k}$. Then there is a topological representation of $D$ on $S^{n+k}$, which is given by the representation $\alpha$ on a standardly embedded $S^{k-1}$, and away from this $S^{k-1}$ may be equivariantly identified with the restriction of $D \times{ }_{\alpha} Z^{k}$ to $D$ acting on $S^{n} \times R^{k}$, hence is free off $S^{k-1}$.

Proof. Consider as in Lemma 5.2 the manifold $M=\left(S^{n} \times R^{k}\right) / Z^{k}$, and the map $M \rightarrow T^{k}$. Up to homotopy this is a spherical fibration. We replace this spherical fibration by a block fibration $\bar{M} \rightarrow T^{k}$, so $\bar{M}$ is compact. We have 
a homotopy commutative diagram

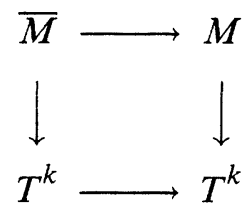

and $\bar{M} \rightarrow M$ is a homotopy equivalence. The universal cover of $\bar{M}$ is a block fibration over $R^{k}$, so it is block (and hence boundedly) equivalent to the trivial block fibration $S^{n} \times R^{k} \rightarrow R^{k}$.

We thus have a bounded homotopy equivalence $f: \widetilde{M} \rightarrow S^{n} \times R^{k}$ with respect to the projection on $R^{k}$. It is easy to see this map is almost $Z^{k}$-equivariant with respect to the standard action of the second factor of $S^{n} \times R^{k}$. Using the bounded surgery theory [FP2], it is easy to see that there is only one element in the bounded structure set of $S^{n} \times R^{k} \rightarrow R^{k}$. (Crossing with $R$ induces isomorphism on simply connected $L$-groups, and at normal space level.) Hence there is a bounded homotopy of $f$ to a homeomorphism $h$. On $\widetilde{M}$, we have the free action of $D \times{ }_{\alpha} Z^{k}$, and we want to consider the conjugate action by $h$ on $S^{n} \times R^{k}$. First we notice this action restricted to $Z^{k}$ makes the projection $p$ to $R^{k}$ almost equivariant. To see this consider

$$
d\left(p h z h^{-1} x, z p x\right) \leq d\left(p h\left(z h^{-1} x\right), p f\left(z h^{-1} x\right)\right)+d\left(p f z h^{-1} x, z p x\right) .
$$

The first term is bounded since $f$ is boundedly homotopic to $h$. The second term is a bounded distance from $d\left(z p f h^{-1} x, z p x\right)$, since $f$ and $p$ are almost equivariant with respect to the standard action on the second factor. Since $z$ is an isometry of $R^{k}$, this is equal to $d\left(p f h^{-1} x, p x\right)$ which is bounded since $f$ is boundedly homotopic to $h$. We now consider this conjugate action on $S^{n} \times R^{k}$, and we want to show the projection to $R^{k}$ is almost equivariant with respect to the $\left(D \times_{\alpha} Z^{k}\right)$-action obtained by letting $D$ act on $R^{n}$ by the representation. Choose $U \subseteq S^{n} \times R^{k}$, compact so that $\bigcup_{z \in Z^{k}} z \cdot U=S^{n} \times R^{k}$. Replacing $U$ by $\bigcup_{g \in D} g \cdot U$ we may assume $U$ to be $D$-invariant. Note that since $D$ is finite and $U$ is compact, $\bigcup_{g \in D} g \cdot p(U)$ must have finite diameter.

Consider $x \in S^{n} \times R^{k}$ and $g \in D$. By the choice of $U$, there is $u \in U$ and $z \in Z$ so that $x=z \cdot u$. Now

$$
\begin{aligned}
d(p g x, g p x) & =d(p g z u, g p z u) \\
\leq & d\left(p g z u, g z g^{-1} p g u\right)+d\left(g z g^{-1} p g u, g z p u\right)+d(g z p u, g p z u) .
\end{aligned}
$$

The first term is $d\left(p g z u, g z g^{-1} p g u\right)=d\left(p\left(g z g^{-1}\right) g u, g z g^{-1} p g u\right)$, and this is already shown to be bounded since $g z g^{-1} \in Z^{k}$. The second term is $d\left(g z g^{-1} p g u, g z p u\right)=d\left(g z g^{-1} p g u, g z g^{-1} g p u\right)=d(p g u, g p u)$ since $g z g^{-1} \in Z^{k}$ acts as isometry on $R^{k}$, but $g p u$ and $p g u$ both belong to $\bigcup g p(U)$, which has finite diameter. Finally, the third term is $d(g z p u, g p z u)=$ $d(z p u, p z u)$ since $g$ acts by isometry, and this term is bounded since $z \in Z^{k}$. 
We are now ready to construct the action of $D$ on $S^{n+k}$. Identifying $S^{n+k}-$ $S^{k-1}$ with $S^{n} \times R^{k}$ using the join lines, one sees that a homeomorphism bounded in the $R^{k}$-factor will be small in $S^{n+k}$ when we approach $S^{k-1}$, hence defining the action on $S^{n+k}$ by the representation on $S^{k-1}$. Using the abovementioned identification with $S^{n} \times R^{k}$ away from $S^{k-1}$ produces a continuous action on $S^{n+k}$.

\section{THE TRANSFER}

Given a linear representation $V$ of a finite group $G$ and exhibiting $V$ with a bounded equivariant triangulation, tensoring with the cellular chains of $V$ defines a natural transformation from $\mathscr{C}_{p t}(R G)=\mathscr{C}_{p t, G}(R)$ to the category of chain complexes in $\mathscr{C}_{V, G}(R)$, and hence from

$$
K_{*}\left(\mathscr{C}_{p t}(R G)\right) \longrightarrow K_{*}\left(\mathscr{C}_{V, G}(R)\right),
$$

preserving the involution when $\operatorname{dim}(V)$ is even and sending the involution to minus the involution when $\operatorname{dim}(V)$ is odd. Using [R1], we get, similarly, maps from

$$
L_{n}^{h}(Z G)=L_{n}^{h}\left(\mathscr{C}_{p t, G}(R)\right) \longrightarrow L_{n+\operatorname{dim}(V)}^{h}\left(\mathscr{C}_{V, G}(R)\right)
$$

and these maps fit into Ranicki-Rothenberg exact sequences as follows:

$$
\begin{array}{cccc}
\vdots & & \vdots \\
\downarrow & & \\
\widehat{H}^{0}\left(Z_{2}, \widetilde{K}_{0}(R G)\right) & \longrightarrow & \widehat{H}^{0+\operatorname{dim}(V)}\left(Z_{2}, \widetilde{K}_{0}(R G)\right) \\
\downarrow & & \downarrow \\
L_{3}^{h}(R G) & \longrightarrow & L_{3+\operatorname{dim}(V)}^{h}\left(\mathscr{C}_{V, G}(R)\right) \\
\downarrow & & \downarrow \\
L_{3}^{p}(R G) & \longrightarrow & L_{3+\operatorname{dim}(V)}^{p}\left(\mathscr{C}_{V, G}(R)\right) \\
\downarrow & & & \\
\widehat{H}^{1}\left(Z_{2}, \widetilde{K}_{0}(R G)\right) & \longrightarrow & \widehat{H}^{1+\operatorname{dim}(V)}\left(Z_{2}, \widetilde{K}_{0}(R G)\right) \\
\downarrow & & & \downarrow
\end{array}
$$

These transfers correspond geometrically to the twisted product with $V$. Consider the following diagram:

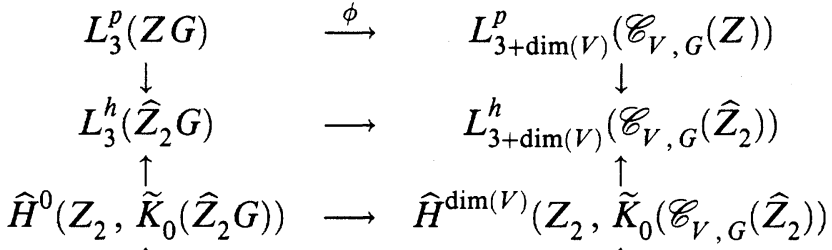

$$
\begin{aligned}
& L_{0}^{p}\left(\widehat{Z}_{2} G\right) \quad \longrightarrow \quad L_{\operatorname{dim}(V)}^{p}\left(\mathscr{\mathscr { C }}_{V, G}\left(\widehat{Z}_{2}\right)\right) \\
& L_{0}^{h}\left(\widehat{Z}_{2} G\right) \quad \longrightarrow \quad L_{\operatorname{dim}(V)}^{h}\left(\mathscr{C}_{V, G}\left(\hat{Z}_{2}\right)\right)
\end{aligned}
$$


where the horizontal maps are the transfers induced by $V$, the lower vertical maps are pieces of Ranicki-Rothenberg exact sequences, and the map $L_{3}^{p}(Z G) \rightarrow L_{3}^{h}\left(\widehat{Z}_{2} G\right)$ is induced by $Z \rightarrow \hat{Z}_{2}$ and the fact that $\widetilde{K}_{0}(Z G) \rightarrow$ $\widetilde{K}_{0}\left(\widehat{Z}_{2} G\right)$ is trivial for $G$ a finite group [S1]. $L_{3}^{p}\left(\mathscr{C}_{V, G}(Z)\right) \rightarrow L_{3}^{h}\left(\mathscr{C}_{V, G}\left(\widehat{Z}_{2}\right)\right)$ is defined when $\widetilde{K}_{0}\left(\mathscr{C}_{(V, G)}(Z)\right) \rightarrow \widetilde{K}_{0}\left(\mathscr{C}_{(V, G)}\left(\hat{Z}_{2}\right)\right)$ is trivial, which is the case when $G$ is the dihedral group. It is our aim to study the map labelled $\phi$ in diagram (6.2) on elements in $L_{3}^{p}(Z G)$, mapping nontrivially to $L_{3}^{h}\left(\widehat{Z}_{2} G\right)$, where the nontriviality is detected by an element in $H^{0}\left(Z_{2}, \widetilde{K}_{0}\left(\widehat{Z}_{2} G\right)\right)$, using the fact that $L_{0}^{h}\left(\widehat{Z}_{2} G\right) \rightarrow L_{0}^{p}\left(\widehat{Z}_{2} G\right)$ is onto.

Let 0 be the 0 -dimensional representation of $G$. The category $\mathscr{C}_{0, G}(R)$ is the same as $\mathscr{C}_{p t}(R G)$ and we have the following:

Theorem 6.3. On $K$-theory the transfer induced by the representation $V$ is given by

$$
\widetilde{K}_{0}(R G)=\widetilde{K}_{0}\left(\mathscr{C}_{0, G}(R)\right) \longrightarrow \widetilde{K}_{0}\left(\mathscr{C}_{V, G}(R)\right),
$$

where the arrow is induced by the inclusion of 0 in $V$.

Proof. Given a projective $R G$-module $P$, represented by a projection $p: F \rightarrow$ $F$, we want to consider the induced projection $F \otimes C_{\#}(V) \rightarrow F \otimes C_{\#}(V)$, where $C_{\#}(V)$ are the cellular chains of a bounded triangulation of $V$. The short exact sequence of chain complexes $0 \rightarrow C_{\#}(0) \rightarrow C_{\#}(V) \rightarrow C_{\#}(V, 0) \rightarrow 0$, where $C_{\#}(0)=R$, shows it is enough to prove that $p: F \otimes C_{\#}(V, 0) \rightarrow F \otimes C_{\#}(V, 0)$ is the trivial element in $\widetilde{K}_{0}\left(\mathscr{C}_{V, G}(R)\right)$. Let $V^{s}=\bigcup_{H \subset G} V^{H}$. We then have

$$
\left[F \otimes C_{\#}(V, 0) ; p\right]=\left[F \otimes C_{\#}\left(V, V^{s}\right) ; p\right]+\left[F \otimes C_{\#}\left(V^{s}, 0\right) ; p\right],
$$

but on $F \otimes C_{\#}\left(V, V^{s}\right)$ the $G$-action is free, so we may use Eilenberg swindle to prove the element is 0 . Working down inductively on orbit types modulo lower strata, we obtain that $\left[F \otimes C_{\#}\left(V^{s}, 0\right), p\right]$ is trivial, so we are done.

\section{THE OBSTRUCTION}

We want to study the transfer of $\S 6$ on an element $\sigma \in L_{3}^{p}(Z D)$ given by a surgery problem $M \rightarrow X$, where $X$ is a Swan complex for the dihedral group. Note that $X$ is finite, so $\sigma$ actually comes from $L_{3}^{h}(Z D) ; X$ is even simple measured in $\mathrm{Wh}(Q \pi)$, so $\sigma$ comes from $L_{3}^{\prime}(Z D)$ [W2, Proposition 7.1]. By abuse of notation we shall denote the element by $\sigma$ in all cases. The nontriviality of $\sigma$ is an $L$-theoretic explanation of the fact, due to Milnor [Mi], that the dihedral group does not act freely on a sphere. This $L$-theoretic description is due to J. Davis [D], who gave an interpretation of R. Lee's semicharacteristic [L]. Here, we will need the fact [HM1, 7.15, 7.18] that $\sigma$ lies in the top component of $L_{3}^{p}(Z D)$, and that its image is nontrivial in the top component of $L_{3}^{h}\left(\widehat{Z}_{2} D\right)$. 
We also need information about $\widetilde{K}_{0}\left(\mathscr{C}_{V, G}(R)\right)$ for $R=\widehat{Z}_{2}, G=D$ the dihedral group of order $2 p, p$ an odd prime, or rather about the map $\widetilde{K}_{0}(R G) \rightarrow$ $\widetilde{K}_{0}\left(\mathscr{C}_{V, G}(R)\right)$ induced by including 0 in $V$. In general this may be studied using a spectral sequence [Q2] but we confine ourselves to the dihedral group case, where the system of subgroups is very simple.

Proposition 7.1. Let $D$ be the dihedral group of order $2 p, p$ an odd prime. Then the transfer $\widetilde{K}_{0}(R D) \rightarrow \widetilde{K}_{0}\left(\mathscr{C}_{V, D}(R)\right)$ is the trivial map if $V$ has a trivial summand. If $V$ has no trivial summand but a nontrivial one-dimensional (flip) summand $R_{\text {_ }}$ then the map is onto and the kernel is the image of $\widetilde{K}_{0}\left(R C_{p}\right)$. If $V$ has no one-dimensional summands, it is an isomorphism.

Proof. By Theorem 6.3 the transfer is the same as the map induced by inclusion of 0 in $V$. To prove the last part first consider $0 \subseteq V^{s} \subseteq V$, where $V^{s}$ is the singular set of the action. Applying $K$-theory to

$$
\mathscr{C}_{V^{s}, D}^{>0}(R) \longrightarrow \mathscr{C}_{V, D}^{>0}(R) \longrightarrow \mathscr{C}_{V, D}^{>V^{s}}(R)
$$

and using that $\mathscr{C}_{V^{s}, D}^{>0}(R)=\mathscr{C}_{V^{C_{2}} / N\left(C_{2}\right)}^{>0}\left(R C_{2}\right)$, then $K_{*}\left(\mathscr{C}_{V^{s}, D}^{>0}(R)\right)$ is unreduced homology $h_{*-1}\left(S V^{C_{2}} / N\left(C_{2}\right) ; \operatorname{Alg} K R C_{2}\right)$ with coefficients in the algebraic $K$ theory spectrum of $R C_{2}$. Since $\widetilde{K}_{i}\left(R C_{2}\right)=0$ when $i \leq 1$ we get $\widetilde{K}_{i}\left(\mathscr{C}_{V^{s}, D}^{>0}(R)\right)$ $=0$ for $i \leq 2$. The point is that we essentially have the fundamental group $C_{2}$, the cyclic group of order 2 . Similarly on $\mathscr{C}_{V, D}^{>V^{s}}(R)$, the action of $D$ on $V$ away from $V^{s}$ is free, so we are essentially simply connected, and so from the exact sequence we get that $\widetilde{K}_{0}(R D) \rightarrow \widetilde{K}_{0}\left(\mathscr{C}_{V, D}(R)\right)$ is an isomorphism.

In case $V$ has a trivial summand, the map factors through $\widetilde{K}_{0}\left(\mathscr{C}_{R}(R D)\right)=$ $K_{-1}(R D)$ and this map is trivial because of an Eilenberg swindle argument. If there are flip factors in the representation or equivalently $V^{C_{p}} \neq 0$ we need to filter $0 \subseteq V^{C_{p}} \subseteq V^{S} \subseteq V$. But $K_{1}\left(\mathscr{C}_{V^{C_{p}, D}}^{>0}\right) \cong \widetilde{K}_{0}\left(Z C_{p}\right)$ by an argument similar to above, and using that $K_{-1}\left(R C_{p}\right)=0$ it is easy to see the map is induced by inclusion, $C_{p} \subseteq D$.

Theorem 7.2. Let $D$ be the dihedral group of order $2 p, p$ an odd prime, and let $V$ be a representation of $D$ containing no one-dimensional factors. Then the transfer

is nontrivial on $\sigma$.

$$
L_{3}^{p}(Z D) \longrightarrow L_{3+\operatorname{dim}(V)}^{p}\left(\mathscr{C}_{V, D}(Z)\right)
$$

Proof. It follows from Proposition 7.1 that the transfer is an isomorphism on $K_{0}$. The result will follow if the element in $\widehat{H}^{0+\operatorname{dim}(V)}\left(Z_{2}, \widetilde{K}_{0}\left(\mathscr{C}_{V, D}\left(\widehat{Z}_{2} G\right)\right)\right.$ is not in the image from $L_{\operatorname{dim}(V)}^{p}\left(\mathscr{C}_{V, D}\left(\widehat{Z}_{2}\right)\right)$. Consider the diagram (6.2). This is a diagram of Mackey functors. Tensoring the diagram with $\hat{Z}_{2}$ preserves exactness since $\widehat{Z}_{2}$ is flat, so we may use Theorem 4.5, or rather Remark 4.6. The projective in question is in the top component. We may of course arrange the 
dimension of $V$ to be divisible by 4 (otherwise compose with two-dimensional transfer). There is a diagram of exact sequences.

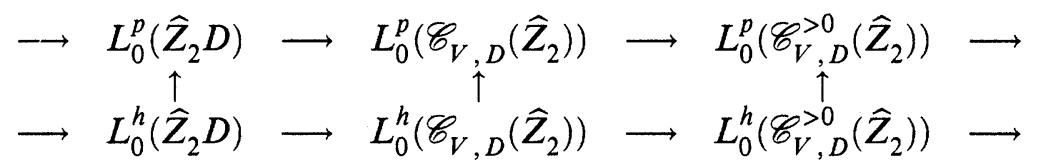

This is because $\widetilde{K}_{i}\left(\mathscr{C}_{V, D}^{>0}\left(\hat{Z}_{2}\right)\right)=0$ for $i \leq 1$, and thus the decorations are as indicated in the diagram. The map $L_{0}^{h}\left(\mathscr{C}_{V, D}^{>0}\left(\widehat{Z}_{2}\right)\right) \rightarrow L_{0}^{p}\left(\mathscr{C}_{V, D}^{>0}\left(\widehat{Z}_{2}\right)\right)$ is thus clearly an isomorphism, but this is not quite enough. However the effective fundamental group is trivial and cyclic of order 2, so on the top component these groups are trivial by Theorem 4.5. This means that on the top component every third group in the sequence is trivial and the fact that $L_{0}^{h}\left(\widehat{Z}_{2} D\right) \rightarrow L_{0}^{p}\left(\widehat{Z}_{2} D\right)$ is onto thus implies the result.

Lemma 7.3. The groups $L_{*}^{p}\left(Z C_{p} \rightarrow Z D\right)$ and $L_{*}^{p}\left(\mathscr{C}_{R_{-}, D}(Z)\right)$ are equal under the map that glues on a product with $[0, \infty)$ to the boundary, and under this identification the $R_{-}$-transfer corresponds to the classical transfer [W1, Chapter 12].

Proof. Comparing the long exact sequences

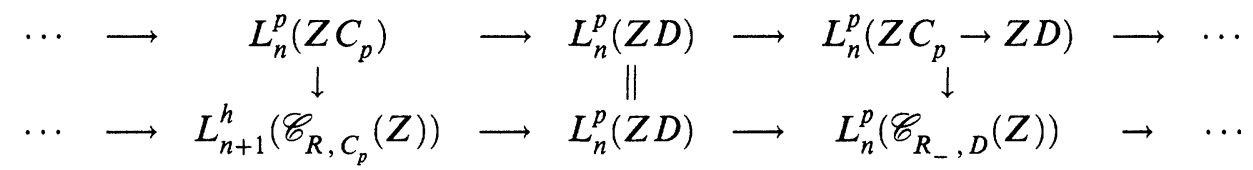

gives the result by using the 5-lemma and the fact that the twisted product restricted to the cyclic subgroup is the untwisted product, which is an isomorphism.

Theorem 7.4. The transfer $L_{3}^{p}(Z D) \rightarrow L_{0}^{p}\left(Z C_{p} \rightarrow Z D\right)$ is nontrivial on $\sigma$.

Proof. The transfer sits in an exact sequence

$$
L N_{3}^{p}\left(Z C_{p} \longrightarrow Z D^{-}\right) \longrightarrow L_{3}^{p}(Z D) \longrightarrow L_{0}^{p}\left(Z C_{p} \longrightarrow Z D^{-}\right)
$$

which by $[\mathrm{H}$, Theorem 3] can be naturally identified with

$$
L_{3}^{p}\left(Z C_{p}, \alpha, 1\right) \longrightarrow L_{3}^{p}(Z D, \alpha, 1) \longrightarrow L_{3}^{p}\left(Z C_{p} \longrightarrow Z D, \alpha, 1\right),
$$

where $\alpha(g)=w(g) \operatorname{tg}^{-1}$ after fixing $t \in D-C_{p}$, all $g \in D$. It is known that the image of $\sigma$ is contained in the top component, and is nontrivial in $L_{3}^{h}\left(\widehat{Z}_{2} D\right) \cong L_{3}^{h}\left(\widehat{Z}_{2} D, \alpha, 1\right)$, so we proceed by looking at $L_{3}^{h}\left(\widehat{Z}_{2} C_{p}, \alpha, 1\right) \stackrel{\text { Ind }}{\longrightarrow}$ $L_{3}^{h}\left(\widehat{Z}_{2} D, \alpha, 1\right)$ on the top component. But this map is zero since the restriction $L_{3}^{h}\left(\widehat{Z}_{2} D, \alpha, 1\right) \stackrel{\text { Res }}{\longrightarrow} L_{3}^{h}\left(\widehat{Z}_{2} C_{p}, \alpha, 1\right)$ is an injection by [HM2, 2.17] and Res.Ind is multiplication by 2 (so induces the zero map). Hence the transfer is nontrivial 2-adically on $\sigma$.

Theorem 7.5. Let $D$ be the dihedral group of order $2 p, p$ an odd prime, and let $V$ be a representation of $D$ containing exactly one one-dimensional factor 
$R_{-}$and no other one-dimensional (trivial) summands. Then the transfer

$$
L_{3}^{p}(Z D) \longrightarrow L_{3+\operatorname{dim}(V)}^{p}\left(\mathscr{C}_{V, D}(Z)\right)
$$

is nontrivial on $\sigma$.

Proof. Let $V=V_{0} \times R_{-}$. As usual assume $V_{0}$ has dimension divisible by 4 . Consider the diagram

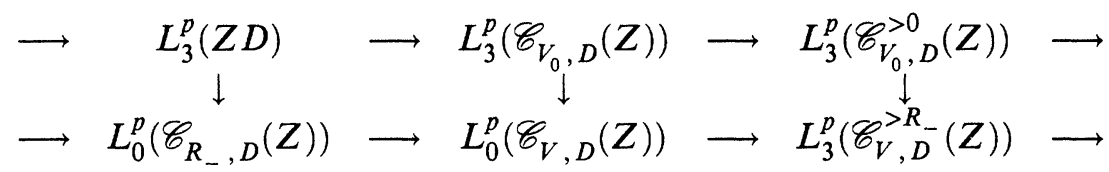

where the horizontal maps are induced by the inclusion $0 \subseteq V_{0}$ and $R_{-} \subseteq$ $V_{0} \times R_{-}=V$. The vertical maps are the $R_{-}$-transfers. Note that by Theorem 4.5 both $L_{3}^{p}\left(\mathscr{C}_{V, D}^{>0}(Z)\right)$ and $L_{3}^{p}\left(\mathscr{C}_{V, D}^{>0}(Z)\right)$ are zero in the top component. Therefore the diagram reduces the final $R_{-}$-transfer to Lemma 7.3.

It turns out to be quite easy to treat the trivial summands of the representation since $K_{-1}(Z D)$ is trivial. We have

Theorem 7.6. Let $D$ be the dihedral group of order $2 p, p$ an odd prime, and let $V$ be a representation of $D$ containing exactly one factor $R_{-}$. Then the transfer

$$
L_{3}^{p}(Z D) \longrightarrow L_{3+\operatorname{dim}(V)}^{p}\left(\mathscr{C}_{V, D}(Z)\right)
$$

is nontrivial on $\sigma$.

Proof. The only remaining question is when $V$ contains trivial summands. Write $V=U \times W$, where $W$ is trivial. The $W$ transfer is simply crossing with $W$ and algebraically this is the map that changes the upper index in the bounded surgery group. But $K_{-1}(Z D)$ trivial implies $K_{-1}\left(\mathscr{C}_{V, D}(Z)\right)$ trivial so the Ranicki-Rothenberg exact sequence gives the result.

We have in the other direction

Theorem 7.7. The transfer associated with $R_{-}^{2}$ from $L_{3}^{h}(Z D) \rightarrow L_{1}^{h}\left(\mathscr{C}_{R_{-}^{2}, D}(Z)\right)$ is trivial on $\sigma$.

Proof. In Lemma 7.3 above we identified the $R_{-}$-transfer with the classical transfer. Using the fact that $\sigma$ comes from $L_{3}^{\prime}(Z D)$ we finish the proof by the following lemma and a diagram chase using the fact that $L_{3}^{\prime}(Z C)=0$.

Lemma 7.8. Let $D=D_{2 p}$ and $C=C_{p}$. Then the transfer

$$
L_{0}^{\prime}\left(Z D^{-}\right) \longrightarrow L_{1}^{\prime}(Z C \longrightarrow Z D)
$$

is zero.

Proof. From Wall's calculations (see [W3, 5.3]), the composite $L_{0}^{\prime}\left(Z D^{-}\right) \rightarrow$ $L_{0}^{\prime}\left(\widehat{Z}_{2} D^{-}\right) \rightarrow L_{0}^{K}\left(\widehat{Z}_{2} D^{-}\right) \cong g_{2}(Z / 2)$ is an isomorphism, where $g_{2}$ is the number of primes in $Z\left[\zeta_{p}+\zeta_{p}^{-1}\right]$ dividing 2 , and we work in the top component 
throughout. The transfer above sits in the following commutative diagram:

$$
\begin{aligned}
& L N_{0}^{\prime}(Z C \longrightarrow Z D) \longrightarrow L_{0}^{\prime}\left(Z D^{-}\right) \longrightarrow L_{1}^{\prime}(Z C \longrightarrow Z D) \\
& L N_{0}^{\prime}\left(\hat{Z}_{2} C \longrightarrow \widehat{Z}_{2} D\right) \longrightarrow L_{0}^{\prime}\left(\hat{Z}_{2}^{\downarrow} D^{-}\right) \longrightarrow L_{1}^{\prime}\left(\widehat{Z}_{2} C \longrightarrow \widehat{Z}_{2} D\right) \\
& L N_{0}^{K}\left(\widehat{Z}_{2} C \longrightarrow \widehat{Z}_{2} D\right) \rightarrow L_{0}^{K}\left(\widehat{Z}_{2} D^{-}\right)
\end{aligned}
$$

By $[H$, Theorem 3] the top horizontal sequence may be naturally identified with $L_{2}^{\prime}(Z C, \alpha, 1) \rightarrow L_{2}^{\prime}(Z D, \alpha, 1) \rightarrow L_{2}^{\prime}(Z C \rightarrow Z D, \alpha, 1)$, where $\alpha(g)=$ $t g^{-1} t^{-1}$ for $g \in D$, after choosing $t \in D-C$. There is a similar identification for the other two rows. But

$$
L_{2}^{K}\left(\widehat{Z}_{2} C, \alpha, 1\right) \longrightarrow L_{2}^{K}\left(\widehat{Z}_{2} D, \alpha, 1\right)
$$

is surjective [HM2, 2.10] and in the left-hand vertical sequence

$$
L_{2}^{\prime}\left(\widehat{Z}_{2} C, \alpha, 1\right)=\widetilde{g}_{2}\{ \pm 1\} \oplus \widetilde{g}_{2}(Z / 2),
$$

where $\widetilde{g}_{2}=\#$ primes dividing 2 in $Z\left[\zeta_{p}\right]$ (see [W3, 4.3] where we are in case $\left.\mathrm{III}_{a}\right)$. The map

$$
L_{2}^{\prime}\left(\widehat{Z}_{2} C, \alpha, 1\right) \longrightarrow L_{2}^{K}\left(\widehat{Z}_{2} C, \alpha, 1\right) \cong \widetilde{g}_{2}(Z / 2)
$$

is just projection on the second factor. Now the map

$$
L_{2}^{\prime}(Z C, \alpha, 1) \longrightarrow L_{2}^{\prime}\left(\widehat{Z}_{2} C, \alpha, 1\right)
$$

is calculated in [W3, 4.5.2] : the image is

$$
\operatorname{ker}\left\{\psi_{2}: L_{2}^{\prime}\left(\widehat{Z}_{2} C, \alpha, 1\right) \longrightarrow L_{2}^{\prime}\left(Z C \longrightarrow \widehat{Z}_{2} C, \alpha, 1\right)\right\} \cong \widetilde{g}_{2}(Z / 2),
$$

the second factor in (7.10). Therefore $L_{2}^{\prime}(Z C, \alpha, 1) \rightarrow L_{2}^{\prime}(Z D, \alpha, 1)$ is surjective, hence the transfer is zero.

Combining the above we get our main theorems.

Theorem 7.11. Let $V$ be a linear representation of the dihedral group of order $2 p, p$ an odd prime. Then there is an action of $D$ on a sphere free off $a$ standard proper subsphere and given by $S(V)$ on the subsphere if and only if the representation has at least two $R_{-}$-factors.

Proof. We have the following diagram of surgery exact sequences where the vertical arrows are transfers associated to $V$ :

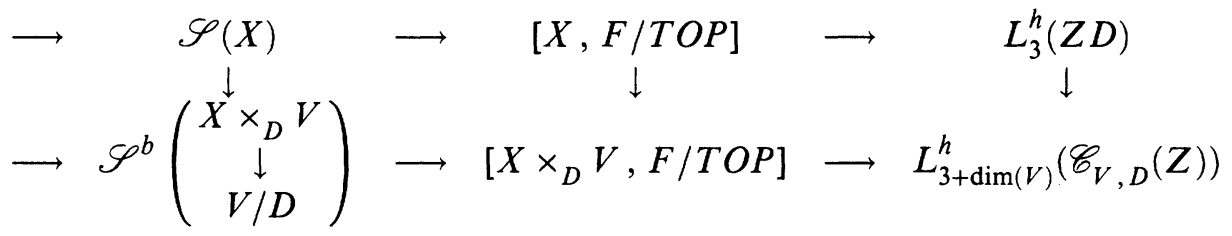

The map $[X, F / T O P] \rightarrow\left[X \times_{D} V, F / T O P\right]$ is an isomorphism since $X \rightarrow$ $X \times{ }_{D} V$ is a homotopy equivalence. It follows from Theorem 2.5 that the 
existence of a group action implies the structure set $\mathscr{S}^{b}\left(\begin{array}{c}X \times_{D} V \\ \downarrow \\ V / D\end{array}\right)$ is nonempty, but this contradicts the nontriviality of the $L$-theory transfer on $\sigma$ of Theorem 7.6. On the other hand when as in Theorem 7.7 the surgery obstruction is zero, then Theorem 2.5 tells us the action does exist.

From Theorem 5.3 we get

Theorem 7.12. If $D \times{ }_{\alpha} Z^{k}$ acts freely, cocompactly, and properly discontinuously on $S^{n} \times R^{m}$ for some $n, m$, then $\alpha$ considered as a real representation has at least two $R_{-}$-factors.

\section{ACTIONS ON $S^{n} \times R^{m}$}

In this section we give examples to show that Theorem 7.12 gives the best possible condition for actions of $D_{p} \times_{\alpha} Z^{k}$. We begin by constructing a minimal example.

Consider $X$ a $(4 l+3)$-dimensional Swan complex for the dihedral group $D$ of order $2 p, p$ an odd prime, and a surgery problem $M \rightarrow X$ in the smooth category. We may assume $X$ is a finite complex, which may even be taken to be simple measured in $\mathrm{Wh}(Q D)$. Consider $T^{2}$ with $D$ acting through the cyclic group of order 2 by conjugation on two $S^{1}$ factors. We then have

Theorem 8.1. For any $l \geq 0$, the surgery obstruction of $\widetilde{M} \times{ }_{D} T^{2} \rightarrow \widetilde{X}^{4 l+3} \times_{D} T^{2}$ is trivial.

It immediately follows that

Theorem 8.2. The twisted product $D \times{ }_{\alpha} Z^{2}$, where the twisting is by -1 on the two factors, acts freely, smoothly, and properly discontinuously on $S^{4 l+3} \times R^{2}$ with compact quotient.

Proof. Let $N$ be a solution to the surgery problem which exists by Theorem 8.1 . The universal cover of $N$ is homeomorphic to $S^{4 l+3} \times R^{2}$ and has an action of $D \times{ }_{\alpha} Z^{2}$ as claimed. This produces a smooth action on $\Sigma^{n} \times R^{k}$, where $\Sigma^{n}$ is a homotopy sphere. To produce a smooth action on $S^{n} \times R^{k}$, it is enough by 3.16 to prove that the covering map from $L_{k}\left(D \times_{\alpha} Z^{k}\right)$ to $L_{k}\left(\mathscr{C}_{R^{k}}(Z)\right)$ is onto. However, since the covering map $L_{0}(Z D) \rightarrow L_{0}(Z)$ is onto, and crossing with $R^{k}$ induces an isomorphism from $L_{0}(Z)$ to $L_{k}\left(\mathscr{C}_{R^{k}}(Z)\right)$, we are done.

Proof of Theorem 8.1. Consider $D$ acting on $T^{2}$ by conjugation on both factors. It is easy to see that $T^{2} / D=T^{2} / C_{2}$ is a 2-sphere. The action of $C_{2}$ has exactly four fixed points and is free off these four fixed points. We may give $T^{2}$ an equivariant handlebody structure by one $C_{2}$-free 0 -handle $H_{0}$, three $C_{2}$-free 1-handles $H_{1}, H_{1}^{\prime}, H_{1}^{\prime \prime}$, and four 2-handles with the action fixing the 
center of the handle. We shall consider

$$
\widetilde{M} \times_{D} T^{2} \longrightarrow \begin{gathered}
\widetilde{X} \times{ }_{D} T^{2} \\
\downarrow \\
\\
T^{2} / D
\end{gathered}
$$

trying to solve the surgery problem in a blocked way over $T^{2} / D=S^{2}$. One of the 1-handles, say $H_{1}$, has the property that $q: T^{2} \rightarrow T^{2} / D$ restricted to $H_{0} \cup H_{1}$ is a trivial double cover. Then $\widetilde{M} \times_{D}\left(H_{0} \cup H_{1}\right) \rightarrow \widetilde{X} \times_{D}\left(H_{0} \cup H_{1}\right)$ is $\bar{M} \times\left(\left(H_{0} \cup H_{1}\right) / D\right) \rightarrow \bar{X} \times\left(\left(H_{0} \cup H_{1}\right) / D\right)$, where $\bar{M} \rightarrow \bar{X}$ is the double cover of $M \rightarrow X$. But we know this surgery problem has a solution by the existence of lens spaces, so let $W$ be a normal bordism from $\bar{M}$ to a homotopy equivalence. Now consider the surgery problem over one of the 2-handles $\mathrm{H}_{2}$. We already have a solution on part of the boundary, so what remains gives an obstruction in $L_{5}^{\prime}\left(Z C_{p} \rightarrow Z D\right)$. This is the obstruction of $\widetilde{M} \times{ }_{\alpha} I^{2} \rightarrow \widetilde{X} \times{ }_{\alpha} I^{2}$ with $W \times I \rightarrow \bar{X} \times I$ glued on to part of the boundary. But this is the same element as the one we obtain by first applying the transfer $-\times_{(-1)} I$ to get an element in $L_{4}^{\prime}\left(Z C_{p} \rightarrow Z D^{-}\right)$, then gluing on $W$ to the boundary to get an element of $L_{4}^{\prime}\left(Z D^{-}\right)$, and then making another twisted product with $I$. By Lemma 7.8 this latter transfer is 0 . In the lowest possible dimension $n=3$, we need the results of Freedman to carry out the surgery. We solve the problem over the other 2-handles similarly, and we are left with a problem over the remaining 1-handles relative to their boundary. But the group $L_{5}^{\prime}\left(Z C_{p}\right)=0$ [W4]. This argument always shows the surgery obstruction is zero, even though one of the steps can only be done topologically when $n=3$.

We conclude by the following

Theorem 8.3. The group $D_{p} \times{ }_{\alpha} Z^{k}$ acts freely and properly discontinuously on $S^{m} \times R^{n}$ for some $n, m$ with compact quotient if and only if $n \equiv 3(4), m=k$, and $\alpha$ considered as a real representation has at least two $R_{-}-$factors.

Proof. Suppose we have an action of $D \times{ }_{\alpha} Z^{k}$ on $S^{n} \times R^{m}$. By Lemma 5.1, $n \equiv 3(4)$, and by Lemma 5.2, $k=m$. By Theorem 7.12, $\alpha$ considered as a real representation must have at least two $R_{-}$-factors. Conversely let $L=\left(Z^{k}, \alpha\right)$ be an integral representation whose associated real representation contains two $R_{-}$-factors. Then there is a projection $L \otimes Q \rightarrow Q_{-} \oplus Q_{-}$, and the image of $L \otimes Z$ must be $Z_{-} \oplus Z_{-}$. Hence we have a $D$-invariant exact sequence $0 \rightarrow L_{0} \rightarrow$ $L \rightarrow Z_{-} \oplus Z_{-} \rightarrow 0$, where $L_{0}=\left(Z^{k-2}, \alpha_{0}\right)$ is an integral subrepresentation of $L$. From this we construct a $D$-equivariant bundle $T^{k-2} \rightarrow T^{k} \rightarrow T^{2}$, where the actions are given by $\alpha_{0}, \alpha$, and -1 respectively. But then we have a bundle $\tilde{X} \times_{\alpha} T^{k} \rightarrow \tilde{X} \times_{(-1)} T^{2}$ and by Theorem 8.1 there is a manifold homotopy equivalent to $\widetilde{X} \times_{(-1)} T^{2}$, so by pullback to the total space, we obtain a manifold homotopy equivalent to $\widetilde{X} \times_{\alpha} T^{k}$. We finish the proof the same way as in Theorem 8.2. 


\section{REFERENCES}

[AH] D. R. Anderson and W.-C. Hsiang, The functors $K_{-i}$ and pseudoisotopies of polyhedra, Ann. of Math. (2) 105 (1977), 201-233.

[AM1] D. R. Anderson and H. J. Munkholm, Boundedly controlled topology, foundations of algebraic topology and simple homotopy theory, Lecture Notes in Math., vol. 1323, Springer, 1988.

[AM2] __, Geometric modules and algebraic K-homology theory, $K$-Theory 3 (1990), 561-602.

[AP] D. R. Anderson and E. K. Pedersen, Semifree topological actions of finite groups on spheres, Math. Ann. 265 (1983), 23-44.

[B] S. Bentzen, The non-existence of tensorproducts for free group actions on spheres, Algebraic Topology (Aarhus 1982), Lecture Notes in Math., vol. 1051, Springer, pp. 8-24.

[CP] F. Connolly and S. Prassidis, Groups which act freely on $R^{m} \times S^{n-1}$, Topology 28 (1989), 133-148.

[D] J. Davis, The surgery semicharacteristic, Proc. London Math. Soc. (3) 47 (1983), 411-428.

[DL] T. tom Dieck and P. Löfler, Verschlingung von Fixpunktmengen in Darstellungsformen. I, Algebraic Topology (Göttingen 1984), Lecture Notes in Math., vol. 1172, Springer, pp. 167-187.

[E] A. Edmonds, Equivariant regular neighborhoods, Transformation Groups, London Math. Soc. Lecture Notes, vol. 26, Cambridge Univ. Press, Cambridge, 1977, pp. 51-69.

[FP1] S. Ferry and E. K. Pedersen, Controlled algebraic $K$-theory (to appear).

[FP2] _-, Epsilon surgery theory, Math. Gottingensis 17 (1990).

[H] I. Hambleton, Projective surgery obstructions on closed manifolds, Algebraic $K$-theory (Oberwolfach 1980), Lecture Notes in Math., vol. 967, Springer, pp. 101-131.

[HM1] I. Hambleton and I. Madsen, Actions of finite groups on $R^{n+k}$ with fixed set $R^{k}$, Canad. J. Math. 38 (1986), 781-860.

[HM2] __ On the discriminants of forms of Arf invariant one, J. Reine Angew Math. 395 (1989), 142-166.

[HTW1] I. Hambleton, L. Taylor, and B. Williams, Detection theorems in $K$-theory and L-theory, J. Pure Appl. Algebra 63 (1990), 247-299.

[HTW2] _ Induction theory, preprint, MSRI, 1990.

[L] R. Lee, Semicharacteristic classes, Topology 12 (1973), 183-199.

[M] I. Madsen, Smooth spherical space forms, Geometric Applications of Homotopy Theory (Evanston, 1977), Lecture Notes in Math., vol. 657, Springer, 1978, pp. 303-352.

[M] J. Milnor, Groups which act on $S^{n}$ without fixed points, Amer. J. Math. 79 (1957), 623-630.

[PW] E. K. Pedersen and C. Weibel, $K$-theory homology of spaces, Algebraic Topology (Proceedings Arcata Topology 1986), Lecture Notes in Math., vol. 1370, Springer, pp. 346-361.

[Q1] F. Quinn, Ends of maps. I, Ann. of Math. (2) 110 (1979), 275-331.

[Q2] — Ends of maps. II, Invent. Math. 68 (1982), 353-424.

[R1] A. A. Ranicki, Additive L-theory, $K$-Theory 3 (1989), 163-195.

[R2] Lower $K$ - and L-theory, Math. Gottingensis 25 (1990).

[Ro] J. Rosenberg, $K$ and $K K$ : Topology and operator algebras, preprint, 1989.

[S1] R. G. Swan, Induced representations and projective modules, Ann. of Math. (2) 71 (1960), 267291.

[S2] _- Periodic resolutions for finite groups, Ann. of Math. (2) 72 (1960), 267-291.

[W1] C. T. C. Wall, Surgery on compact manifolds, Academic Press, 1971. 
[W2] C. T. C. Wall, Norms of units in group rings, Proc. London Math. Soc. (3) 29 (1974), 593-632.

[W3] _ Classification of hermitian forms. VI, Group rings Ann. of Math. (2) 103 (1976), 1-80.

[W4] __, Periodic projective resolutions, Proc. London Math. Soc. (3) 39 (1979), 509-553.

Department of Mathematics and Statistics, McMaster University, Hamilton, ONTARIO L8S 4K1, CANADA

Department of Mathematical Sciences, State University of New York at BinghamTON, Binghamton, NeW YORK 13901 